\title{
BMJ Open Oncological safety of hysteroscopy in the diagnosis of stage I endometrial cancer: protocol for a systematic review and meta-analysis
}

\author{
Yu Xu (D) , ,2 Qian Wen Zhang, ${ }^{1,2}$ Yi Du, ${ }^{1,2}$ Zhao Juan Qin,, ${ }^{1,2}$ Yue Dong He, ${ }^{1,2}$ \\ Ai Zheng (iD) ${ }^{1,2}$
}

To cite: Xu Y, Zhang QW, Du Y, et al. Oncological safety of hysteroscopy in the diagnosis of stage I endometrial cancer: protocol for a systematic review and meta-analysis. BMJ Open 2020;10:e041981. doi:10.1136/ bmjopen-2020-041981

- Prepublication history and additional material for this paper is available online. To view these files, please visit the journal online (http://dx.doi.org/10. 1136/bmjopen-2020-041981).

Received 22 June 2020 Revised 14 November 2020 Accepted 16 November 2020

D Check for updates

(C) Author(s) (or their employer(s)) 2020. Re-use permitted under CC BY-NC. No commercial re-use. See rights and permissions. Published by BMJ.

${ }^{1}$ Department of Obstetrics and Gynecology, West China Second University Hospital, Sichuan University, Chengdu, China ${ }^{2}$ Key Laboratory of Birth Defects and Related Diseases of Women and Children (Sichuan University), Ministry of Education, Chengdu, China

Correspondence to

Dr Ai Zheng;

ZhengAi_WestChina@163.com

\section{ABSTRACT}

Background The oncological safety of diagnostic hysteroscopy in patients with stage I endometrial cancer remains uncertain and conflicting. The aim of the proposed systematic review and meta-analysis is to summarise the available evidence examining the association between diagnostic hysteroscopy and the prognosis of stage I endometrial cancer and to statistically synthesise the results of relevant studies.

Methods and analysis Systematic searches of PubMed/ MEDLINE, Embase, Cochrane Library and Web of Science will be undertaken using prespecified search strategies. Two authors will independently conduct eligible studies selection process, perform data extraction and appraise the quality of included studies. Original case-control studies, cohort studies and randomised controlled trails published in English will be considered for inclusion. The outcomes of interest will be 5-year recurrence-free survival, disease-specific survival and overall survival. Meta-analyses will be performed to calculate pooled estimates.

Ethics and dissemination Our study will be based on published data, and thus there is no requirement for ethics approval. The results will be shared through publication in a peer-reviewed journal and presentations at academic conferences.

PROSPERO registration number CRD42020193696.

\section{INTRODUCTION}

Endometrial cancer is the most common malignancy of the female reproductive system in developed countries. ${ }^{1}$ Of the patients with endometrial cancer, the majority will be diagnosed at stage I or stage II, and 5-year survival rate is as high as $80 \%$ to $90 \%$ in these women. ${ }^{23}$ The main symptom of endometrial cancer is abnormal uterine bleeding, this is typically postmenopausal but may also be intermenstrual or heavy/prolonged periods, and these clinical manifestations can be found in up to 90 percent of patients. ${ }^{45}$

The diagnosis of endometrial cancer is based on histological results of endometrial sampling by office endometrial biopsy, dilation
Strengths and limitations of this study

- This proposed systematic review and meta-analysis is the first one on this topic and will compare survival measures of women with stage I endometrial cancer who underwent either diagnostic hysteroscopy or non-hysteroscopic diagnostic procedures.

- Hysteroscopy is widely used in the diagnosis of early endometrial cancer, any potential associations between diagnostic hysteroscopy and prognosis of patients would have significantly practical implications.

- We minimise the potential reviewer bias by letting two independent reviewers to screen for eligible studies, extract the data and assess the quality of the included studies.

- We only include papers published in English.

- A considered heterogeneity is anticipated between studies because of differences in study design and length of follow-up.

and curettage or diagnostic hysteroscopy and direct endometrial biopsy. Hysteroscopy can provide gynaecologist with visualisation of the uterine cavity and is considered to be the most helpful tool for the evaluation of endometrium in presentation of abnormal uterine bleeding. ${ }^{6}$ According to the study of Garuti, hysteroscopy has high sensitivity, specificity, negative predictive value and positive predictive value of $94.2 \%, 88.8 \%, 96.3 \%$ and $83.1 \%$, respectively, in predicting abnormal or normal endometrial histopathology. ${ }^{7}$ Due to its accuracy, hysteroscopy with endometrial biopsy is highly recommended as the gold standard investigation for abnormal uterine bleeding and this procedure is taking the place of the traditional fractional dilation and curettage. ${ }^{89}$

However, concern exists that the use of distention media and increased intrauterine pressure may facilitate the spread of cancer cells into peritoneal cavity though the fallopian tubes, and thereby, a potential deleterious 
effect on staging and prognosis in cases of endometrial cancer. Although positive peritoneal cytology no longer changes the International Federation of Gynaecology and Obstetrics (FIGO) stages of endometrial cancer, ${ }^{10}$ FIGO still recommends obtaining peritoneal washings during surgery because of the potential for positive peritoneal cytology to compound the effects of other risk factors in early stage endometrial cancer. ${ }^{11}$ There was some evidence to suggest that diagnostic hysteroscopy increase the risk of positive peritoneal cytology. ${ }^{12-16}$ Nevertheless, whether or not the positive peritoneal cytology following a diagnostic hysteroscopy is associated with increased mortality or worsened prognosis in patients with endometrial cancer is inconclusive. ${ }^{17-27}$

To our knowledge, there is no systematic review and/ or meta-analysis available on this topic. The aim of the proposed systematic review and meta-analysis is to summarise the available evidence examining the association between diagnostic hysteroscopy and the prognosis of stage I endometrial cancer. The outcomes of interest will be 5-year recurrence-free survival, disease-specific survival and overall survival.

\section{Population}

Women with stage I endometrial cancer diagnosed by hysteroscopy and direct endometrium sampling or by non-hysteroscopic procedures. The final pathological diagnosis of endometrial cancer was made by pathological examination of the specimen after total hysterectomy, the stage of the disease was determined by results of comprehensive staging surgery and pathological examination according to the FIGO staging for the corresponding period.

\section{Exposures}

Hysteroscopy with endometrial biopsy as a preoperative diagnostic procedure for stage I endometrial cancer.

\section{Comparison}

Patients with the stage I endometrial cancer diagnosed by non-hysteroscopic procedures, for example, curettage and office endometrial biopsy.

\section{Outcomes}

Recurrence-free survival, disease-specific survival and overall survival, defined as the period from the date of the diagnosis to the date of recurrence or the last clinic visit (if alive) or the date of death.

\section{Review question}

Does hysteroscopy as a diagnostic procedure worsen the prognosis of cases with stage I endometrial cancer?

\section{METHODS AND DESIGN}

This protocol was drafted according to the Preferred Reporting Items for Systematic Reviews and Meta-Analysis Protocols checklist. ${ }^{28}$ The proposed systematic review and meta-analysis will be conducted in accordance with the standard guideline of 'Meta-analyses Of Observational Studies in Epidemiology (MOOSE) guidelines ${ }^{29}$, and 'Preferred Reporting Items for Systematic Reviews and Meta-Analyses (PRISMA) ${ }^{30}$.

\section{Search strategy}

The leading author (YX) and corresponding author (AZ) will search four electronic databases (PubMed/ MEDLINE, Embase, Cochrane Library and Web of Science) to identify qualifying studies published from database inception until 30 July 2020. Informed by Medical Subject Headings (MeSH), the following keywords will be used to search the databases mentioned: 'endometrial neoplasm', 'cancer of the endometrium', 'carcinoma of the endometrium', 'endometrial cancer', 'endometrial carcinoma', 'endometrium cancer', 'endometrium carcinoma', 'hysteroscopy', 'hysteroscopic surgery', 'uterine endoscopy', 'uteroscopy', 'diagnostic hysteroscopy' and 'hysteroscopic surgical procedure'. The search terms will be combined using Boolean Logic (AND, OR) where needed. We will restrict our search to human studies and peer-reviewed journal articles published in English. The precise search strategies for one of the databases can be found in the online supplemental material 1. In addition, reference lists of all included studies will be manually searched for any further potentially relevant studies. To ensure that the search is comprehensive, the search will be rechecked by an epidemiologist (YDH).

\section{Study selection}

Retrieved records from literature searches will be entered into the EndNote reference manager (V.X9) in order to categorise, manage, remove duplicates and record titles, abstracts and full-texts. Two independent authors (YX and QZ) will screen all titles and abstracts for potentially relevant studies. The full-texts of the relevant studies will then be retrieved and screened for compliance with eligibility criteria by the same two reviewers. For unpublished studies and abstracts that full-texts are not available, we will contact the authors by email to ask for the relevant data. If consensus on eligibility cannot be achieved, a third author (ZQ) will be consulted. For any articles which do not meet the inclusion criteria, the reasons for rejection will be noted. A MOOSE flow diagram documenting the process of study selection will be completed.

\section{Inclusion criteria}

1. Case-control studies, cohort studies or randomised controlled trails.

2. Only English language studies published from inception of databases to 30 July 2020 will be considered.

3. Data must be from an original study.

4. Peer-reviewed papers only will be included.

5. Studies that provide measures of association between diagnostic hysteroscopy and prognosis of patients with stage I endometrial cancer.

\section{Exclusion criteria}

1. Non-human studies. 
2. Paper that are not in English.

3. Case reports, case series, letters, commentaries, notes and editorials.

4. Studies that have include patients of stage II, III and IV endometrial cancer.

5. Only the latest or the most informative study will be included when there are multiple studies that report on the same study population.

6. Abstracts and unpublished studies for which the attempts to contact the authors to get relevant data failed.

\section{Data extraction}

Data from all eligible studies will be independently extracted by two reviewers (YX and YD) using a standardised data collection form, including the name of the first author, year of publication, geographical location, study style, number of centre, number of participant, study span, the duration of follow-up, the outcome(s) of interest, the definition used for each outcome, the confounders adjusted for (if any) and the crude and adjusted measures of association. In cases of relevant papers in which the required data were not reported, the corresponding authors of these studies will be contacted by email to obtain information needed relating to effect estimates. If discrepancies arise in data extraction, these will be discussed between reviewers, and when necessary, a third reviewer (AZ) will be consulted to achieve consensus.

\section{Quality appraisal of included studies}

The quality of all included studies will be independently assessed by two reviewers (YX and YD) using the Risk of Bias Instrument for Non-Randomized Studies of Exposures (ROBINS-E) or the Cochrane collaboration's tool for assessing risk of bias according to the style of the included studies. For each included study, the overall likelihood of bias will be appraised and reported.

The ROBINS-E has seven domains evaluating the source of bias: confounding, selection of participant, classification of the exposures, deviation from intended exposures, missing data, measurement of outcomes and selection of the reported result. ${ }^{31}$ Each domain will be assessed as low, moderate, serious or critical risk of bias, and the study will be rated overall as at least the same level of severity of the highest risk of bias of an individual domain. ${ }^{31}$

For the randomised controlled trails, the risk of bias was assessed by answering the questions about the following features of studies with 'Yes' (low risk of bias), 'No' (high risk of bias) or 'Unclear' (lack of information or uncertainty over the potential bias): random sequence generation, allocation concealment, blinding of participants, blinding of outcome assessment, incomplete outcome data, selective reporting and other bias. ${ }^{32}$ Possible sources of 'other bias' were determined by consensus of the investigators.
Where disagreement in quality appraisal arise, a third opinion from YDH will be obtained.

\section{Data synthesis and assessment of heterogeneity}

Separate meta-analysis will be undertaken for each of the outcomes if possible. Each meta-analysis will be performed to calculate the pooled estimate of the relationship between the diagnostic hysteroscopy and the outcomes. For example, for recurrence-free survival as one of the outcomes of interest, a meta-analysis will be undertaken to investigate the association between the recurrence-free survival and diagnostic hysteroscopy. We will stratify eligible studies into two categories based on the study design: observational study and randomised controlled trail because of the concern that there may be considerable heterogeneity between different types of study. We will perform subgroup analysis according to the type of study and for all outcomes.

Both the crude and adjusted effect estimates will be displayed using the generic inverse variance method. Adjustment will be based on the definition outlined in each of the eligible studies. Heterogeneity among the studies will be assessed by the $\mathrm{x}^{2}$ test and $\mathrm{I}^{2}(<25 \%$ deemed low, $25 \%$ to $50 \%$ deemed moderate and $>50 \%$ deemed high) statistics. $\mathrm{P}$ value $<0.10$ or $\mathrm{I}^{2}>50 \%$ indicates that heterogeneity existed among the studies, so a randomeffects model (Mantel-Haenszel method) will be used. If studies cannot be meaningfully combined in a metaanalysis, they will be presented in tabular format.

Where 10 or more studies are included in a metaanalysis, we will assess the publication bias. The trim and fill method will be used to identify and correct for funnel plot asymmetry arising from publication bias, if appropriate. ${ }^{33}$

\section{Ethics and dissemination}

Our study will be based on published data, and thus there is no requirement for ethics approval. The results will be shared through publication in a peer-reviewed journal and presentations at academic conferences.

\section{Patient and public involvement}

Patients were not involved in the design of this study. However, the authors will communicate the study findings to patient and public groups with interest in this area.

\section{Potential limitations}

There are a number of limitations we can predict in this review. A degree of heterogeneity is anticipated between studies. Differences in the length of follow-up and the study design are the main source for the heterogeneity, and differences in sampling frames are also likely to cause heterogeneity. So, a random-effects model will be used for meta-analyses if there is moderate or high heterogeneity among the included studies.

In all observational studies, the existence of selection bias and residual confounding is a concern. Potential confounders may include age, race, socioeconomic status, degree of histological differentiation, histological type, 
lymphovascular space invasion, pelvic lymph node dissection, para-aortic lymph node dissection, adjuvant chemotherapy and adjuvant radiotherapy. Where possible, our meta-analysis will show both crude and adjusted results, adjusted according to the definitions outlined in each individual study. However, given that less adjusted effect estimates may distort the overall results, a sensitivity analysis will be performed where possible, to examine for more fully adjusted effect estimates for confounders (ie, adjusted for, at a minimum, age, degree of histological differentiation, histological type, lymphovascular space invasion, pelvic lymph node dissection, para-aortic lymph node dissection, adjuvant chemotherapy and adjuvant radiotherapy).

Due to limited resources, only studies which were published in English will be included. Besides, considering that there are many challenges and difficulties to conduct randomised control studies to investigate the oncological safety of hysteroscopy in the diagnosis of stage I endometrial cancer in real clinical settings, the majority of included studies will be observational studies, and this will compromise the results of our proposed study.

\section{DISCUSSION}

There is a lack of consensus on whether diagnostic hysteroscopy deteriorates the prognosis of the early stage endometrial cancer. This proposed systematic review and meta-analysis will summarise the available evidence which has examined these associations, thus providing novel information on the role of hysteroscopy in the evaluation of abnormal uterine bleeding and the diagnosis of endometrial cancer.

Contributors YX, QZ, YD, ZQ, YDH and AZ conceived and designed the protocol, and $Y X$ drafted the protocol manuscript. QZ developed the search strategy, with input from YX, YD and ZQ. YX and YD planned the data extraction. YX and YD planned the quality appraisal of all included studies. YX, QZ, YD, ZQ, YDH and AZ critically revised the manuscript for methodological and intellectual content. All authors approved the final version.

Funding The authors have not declared a specific grant for this research from any funding agency in the public, commercial or not-for-profit sectors.

Competing interests None declared.

Patient consent for publication Not required.

Provenance and peer review Not commissioned; externally peer reviewed.

Supplemental material This content has been supplied by the author(s). It has not been vetted by BMJ Publishing Group Limited (BMJ) and may not have been peer-reviewed. Any opinions or recommendations discussed are solely those of the author(s) and are not endorsed by BMJ. BMJ disclaims all liability and responsibility arising from any reliance placed on the content. Where the content includes any translated material, BMJ does not warrant the accuracy and reliability of the translations (including but not limited to local regulations, clinical guidelines, terminology, drug names and drug dosages), and is not responsible for any error and/or omissions arising from translation and adaptation or otherwise.

Open access This is an open access article distributed in accordance with the Creative Commons Attribution Non Commercial (CC BY-NC 4.0) license, which permits others to distribute, remix, adapt, build upon this work non-commercially, and license their derivative works on different terms, provided the original work is properly cited, appropriate credit is given, any changes made indicated, and the use is non-commercial. See: http://creativecommons.org/licenses/by-nc/4.0/.
ORCID iDs

Yu Xu http://orcid.org/0000-0001-9819-1965

Ai Zheng http://orcid.org/0000-0002-3403-9101

\section{REFERENCES}

1 Siegel RL, Miller KD, Jemal A. Cancer statistics, 2018. CA Cancer J Clin 2018;68:7-30.

2 Lewin SN, Herzog TJ, Medel NIB, et al. Comparative performance of the 2009 International Federation of gynecology and Obstetrics' staging system for uterine corpus cancer. Obstet Gynecol 2010;116:1141-9.

3 Creasman WT, Odicino F, Maisonneuve P, et al. Carcinoma of the corpus uteri. FIGO 26th annual report on the results of treatment in gynecological cancer. Int J Gynaecol Obstet 2006;95:S105-43.

4 Seebacher V, Schmid M, Polterauer S, et al. The presence of postmenopausal bleeding as prognostic parameter in patients with endometrial cancer: a retrospective multi-center study. BMC Cancer 2009;9:460

5 Kimura T, Kamiura S, Yamamoto T, et al. Abnormal uterine bleeding and prognosis of endometrial cancer. IntJ Gynaecol Obstet 2004;85:145-50.

6 Clark TJ, Voit D, Gupta JK, et al. Accuracy of hysteroscopy in the diagnosis of endometrial cancer and hyperplasia: a systematic quantitative review. JAMA 2002;288:1610-21.

7 Garuti G, Sambruni I, Colonnelli M, et al. Accuracy of hysteroscopy in predicting histopathology of endometrium in 1500 women. $J$ Am Assoc Gynecol Laparosc 2001;8:207-13.

8 Revel A, Shushan A. Investigation of the infertile couple: hysteroscopy with endometrial biopsy is the gold standard investigation for abnormal uterine bleeding. Hum Reprod 2002;17:1947-9.

9 de Wit AC, Vleugels MP, de Kruif JH. Diagnostic hysteroscopy: a valuable diagnostic tool in the diagnosis of structural intra-cavital pathology and endometrial hyperplasia or carcinoma?. six years of experience with non-clinical diagnostic hysteroscopy. Eur J Obstet Gynecol Reprod Biol 2003;110:79-82.

10 Pecorelli S. Revised FIGO staging for carcinoma of the vulva, cervix, and endometrium. Int J Gynaecol Obstet 2009;105:103-4.

11 Wethington SL, Barrena Medel NI, Wright JD, et al. Prognostic significance and treatment implications of positive peritoneal cytology in endometrial adenocarcinoma: unraveling a mystery. Gynecol Oncol 2009;115:18-25

12 Chang Y-N, Zhang Y, Wang Y-J, et al. Effect of hysteroscopy on the peritoneal dissemination of endometrial cancer cells: a metaanalysis. Fertil Steril 2011;96:957-61.

13 Polyzos NP, Mauri D, Tsioras S, et al. Intraperitoneal dissemination of endometrial cancer cells after hysteroscopy: a systematic review and meta-analysis. Int J Gynecol Cancer 2010;20:261-7.

14 Obermair A, Geramou M, Gucer F, et al. Does hysteroscopy facilitate tumor cell dissemination? Incidence of peritoneal cytology from patients with early stage endometrial carcinoma following dilatation and curettage (D \& C) versus hysteroscopy and D \& C. Cancer 2000;88:139-43.

15 Zerbe MJ, Zhang J, Bristow RE, et al. Retrograde seeding of malignant cells during hysteroscopy in presumed early endometrial cancer. Gynecol Oncol 2000;79:55-8.

16 Bradley WH, Boente MP, Brooker D, et al. Hysteroscopy and cytology in endometrial cancer. Obstet Gynecol 2004;104:1030-3.

17 Ben-Arie A, TAMIR S, DUBNIK S, et al. Does hysteroscopy affect prognosis in apparent early-stage endometrial cancer? Int J Gynecol Cancer 2008;18:813-9.

18 Cicinelli E, Tinelli R, Colafiglio G, et al. Risk of long-term pelvic recurrences after fluid minihysteroscopy in women with endometrial carcinoma: a controlled randomized study. Menopause 2010;17:511-5

19 Gu M, Shi W, Huang J, et al. Association between initial diagnostic procedure and hysteroscopy and abnormal peritoneal washings in patients with endometrial carcinoma. Cancer 2000;90:143-7.

20 Juhasz-Böss I, Fehm T, Nauth A, et al. Number of hysteroscopies and the time interval between hysteroscopy and surgery: influence on peritoneal cytology in patients with endometrial cancer. Anticancer Res 2010;30:2425-30.

21 Kudela M, Pilka R. Is there a real risk in patients with endometrial carcinoma undergoing diagnostic hysteroscopy (HSC)? Eur J Gynaecol Oncol 2001;22:342-4.

22 Sáinz de la Cuesta R, Angel Espinosa J, Crespo E, et al. Does fluid hysteroscopy increase the stage or worsen the prognosis in 
patients with endometrial cancer? Eur J Obstet Gynecol Reprod Biol 2004:115:211-5.

23 Chen J, Clark LH, Kong W-M, et al. Does hysteroscopy worsen prognosis in women with type II endometrial carcinoma? PLoS One 2017; 12:e0174226.

24 Zhu HL, Liang XD, Wang JL, et al. Hysteroscopy and directed biopsy in the diagnosis of endometrial carcinoma. Chin Med J 2010;123:3524-8.

25 Namazov A, Gemer O, Helpman L, et al. The oncological safety of hysteroscopy in the diagnosis of early-stage endometrial cancer: an Israel gynecologic Oncology Group study. Eur J Obstet Gynecol Reprod Biol 2019;243: :120-4.

26 Soucie JE, Chu PA, Ross S, et al. The risk of diagnostic hysteroscopy in women with endometrial cancer. Am J Obstet Gynecol 2012;207:71.e1-71.e5.

27 Obermair A, Geramou M, Gücer F, et al. Impact of hysteroscopy on disease-free survival in clinically stage I endometrial cancer patients. Int J Gynecol Cancer 2000;10:275-9.
28 Moher D, Shamseer L, Clarke M, et al. Preferred reporting items for systematic review and meta-analysis protocols (PRISMA-P) 2015 statement. Syst Rev 2015;4:1.

29 Stroup DF, Berlin JA, Morton SC, et al. Meta-analysis of observational studies in epidemiology: a proposal for reporting. meta-analysis of observational studies in epidemiology (moose) group. JAMA 2000;283:2008-12.

30 Liberati A, Altman DG, Tetzlaff J, et al. The PRISMA statement for reporting systematic reviews and meta-analyses of studies that evaluate health care interventions: explanation and elaboration. Ann Intern Med 2009;151:W65-94.

31 Hinneburg I. ROBINS-1: a tool for asssessing risk of bias in nonrandomised studies of interventions. Med Monatsschr Pharm 2017;40:175-7.

32 Higgins JPT, Altman DG, Gotzsche PC, et al. The Cochrane Collaboration's tool for assessing risk of bias in randomised trials. BMJ 2011;343:d5928.

33 Weinhandl ED, Duval S. Generalization of TRIM and fill for application in meta-regression. Res Syn Meth 2012;3:51-67. 